(LC-MS/MS). Dysregulated lipids will be correlated with familial risk of depression in samples of G3. RESULTS/ANTICIPATED RESULTS: We hypothesize that dysregulation of lipids and lipid metabolism will be apparent in biospecimens from the high risk compared to the low risk of depression. Also, alterations in RNA transcriptomics of genes involved in lipid metabolic networks are associated with familial risk of depression. Several differential lipid species were previously identified to be associated with MDD. Reduced phosphatidylcholine(PC), phosphatidylethanolamine(PE), phosphatidylinositol(PI), and increased LysoPC, LysoPE, ceramide, triacylglycerol, and diacylglycerol levels have been correlated to MDD. However, these results need to be replicated in independent studies using lipidomics analysis. DISCUSSION/SIGNIFICANCE OF FINDINGS: It is highly likely that completely novel cellular targets will emerge from these studies by uncovering the convergence of lipidomics and genetic variance of lipid metabolic enzymes as biomarkers for predisposition to MDD as well as potential targets for therapeutic development for MDD.

78511

\section{Synthesis of Novel Core/Shell Polymeric Nanoparticles for Controlled Drug Release}

Braden Hahn

Auburn University Samuel Ginn College of Engineering

ABSTRACT IMPACT: This work will develop a novel drug delivery system that has improved biocompatibility and controlled release than current systems and allow for customizable loading and drug delivery to unique patient and treatment requirements. OBJECTIVES/GOALS: The goal of my project is a novel hybrid core/shell nanoparticle system for controlling in vivo chemotherapeutic concentration. The current goal is to confirm core and shell polymeric nanoparticle formation via emulsion technique and validate predictive model developed to optimize shell formation efficiency and control shell thickness. METHODS/STUDY POPULATION: Though early results are promising, they are not proof that the desired core/shell structure is being formed via my novel process. I constructed a theoretical model to use to optimize and control the process for precise shell thicknesses. Therefore, the current experimental plan focus is to not only visually confirm the predicted formation of my core/shell design but use these experiments to validate the model.

1. Gel-Suspended SEM: nanoparticles suspended in gel matrix, bisected to reveal inner structure

2. Fluorescent Conjugation Microscopy: visually-distinct dyes used to show polymer distribution and validated against the theoretical model predictions.

3. Modified Hydrophobic Dye Release: different mixtures of polymers with release showing if previous promising results due to core/shell structure RESULTS/ANTICIPATED RESULTS: As stated, the experiments will confirm the core/shell nanoparticle structure, validate the developed theoretical model, or provide direct evidence against any formation. This core/shell structure is key to the current design for controlling payload release rate and thus in vivo drug concentration. For the gel-suspension experiment, the interior core will be labeled with ultrasmall SPIONs and thus any layers within the particles will be distinct. While this result is qualitative, high magnification fluorescent microscope images will be analyzed using image processing software to determine core/shell formation efficiency and compared to estimated efficiencies from the model. Finally, the mixed release will clarify previous experiments' release mechanism and either support or disprove shell influence. DISCUSSION/ SIGNIFICANCE OF FINDINGS: The significance of this work is twofold: core/shell particles have been proven to provide variable control of release on the micron scale but not yet at the nanoscale, allowing for a circulating, targeted system that can finely control release. The process is also novel for producing this type of structure, at highly consistent quality and size.

81030

\section{Utilizing a synergistic drug combination to target relapsed/refractory FLT3 mutant AML}

LaQuita Jones ${ }^{1}$, Katelyn Melgar ${ }^{1}$, Scott Hoyt ${ }^{2}$, Mark Wunderlich ${ }^{1}$, Eric O'Brien ${ }^{1}$, John Perentesis ${ }^{1}$, Craig Thomas $^{2}$ and Daniel

Starczynowski ${ }^{1}$

${ }^{1}$ Cincinnati Children's Hospital Medical Center; Katelyn Melgar;

${ }^{2}$ National Center for Advancing Translational Sciences

ABSTRACT IMPACT: This work will help to understand a novel therapeutic approach to a common type of acute myeloid leukemia. OBJECTIVES/GOALS: FMS-like tyrosine kinase 3 (or FLT3) mutations occur in $\sim 30 \%$ of acute myeloid leukemia (AML) cases. FLT3 tyrosine kinase domain (TKD) mutations are particularly important in relapsed/refractory FLT3 mutant AML, which portends poor prognosis. This study describes a therapeutic approach to overcoming resistance conferred by FLT3-TKD mutations. METHODS/ STUDY POPULATION: To understand the efficacy of a novel type 1 FLT3 inhibitor (NCGC1481), as a monotherapy and combination therapy, several assays were utilized to interrogate functionality of these therapies. Cell lines and patient samples containing aspartate 835 to tyrosine mutations (D835Y, the most common TKD alteration) and phenylalanine 691 to leucine (F691L) were utilized to examine the effects of NCGC1481 with and without other targeted therapies like MEK inhibitors. Specifically, assays measuring viability, cell death using flow cytometry, in vitro clonogenicity, cellular signaling, and xenograft survival were examined in these FLT3-TKD AML models. Synergy was also measured using well described methods, which also allowed for appropriate dose finding for drug combination experiments. RESULTS/ANTICIPATED RESULTS: Our novel type 1 FLT3 inhibitor (NCGC1481) was particularly effective in the most common FLT3 TKD mutant, D835Y. NCGC1481 reduced viability and cell signaling, while also inducing cell death and prolonging xenograft survival in the FLT3-D835Y model system. In contrast, clinically approved FLT3 inhibitors were less effective at suppressing AML cells expressing FLT3-D835Y. In the case of FLT3-F691L, most of the FLT3 inhibitors tested, including NCGC1481, suppressed canonical FLT3 signaling, but did not significantly reduce viability or leukemic clonogenicity. However, when NCGC1481 was combined with other targeted agents like MEK inhibitors, at synergistic doses, eradication of the FLT3F691L AML clone was substantially increased. DISCUSSION/ SIGNIFICANCE OF FINDINGS: In AML, response to FLT3 inhibitor therapy is often short-lived, with resistance sometimes occurring via FLT3-TKD mutations. Given the dismal prognosis of relapsed FLT3 mutant AML, novel therapies are necessary. This study describes efficacy of a novel FLT3 inhibitor, along with its synergistic activity when combined with other targeted agents. 